\title{
On the dynamical nature of Saturn's North Polar hexagon
}

\author{
Masoud Rostami, Vladimir Zeitlin, Aymeric Spiga
}

\section{To cite this version:}

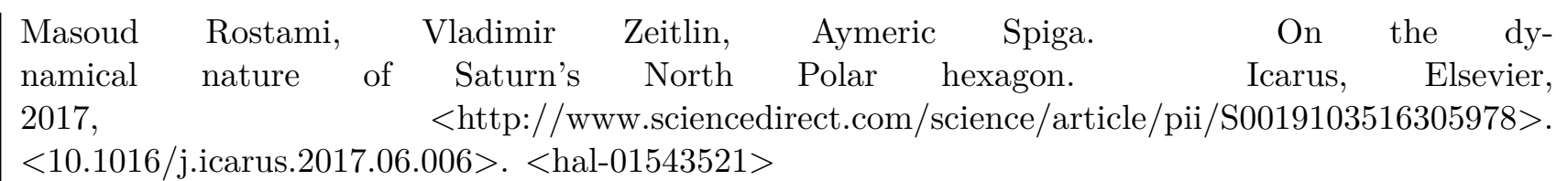

\section{HAL Id: hal-01543521}

http://hal.upmc.fr/hal-01543521

Submitted on 21 Jun 2017

HAL is a multi-disciplinary open access archive for the deposit and dissemination of scientific research documents, whether they are published or not. The documents may come from teaching and research institutions in France or abroad, or from public or private research centers.
L'archive ouverte pluridisciplinaire HAL, est destinée au dépôt et à la diffusion de documents scientifiques de niveau recherche, publiés ou non, émanant des établissements d'enseignement et de recherche français ou étrangers, des laboratoires publics ou privés. 


\section{On the dynamical nature of Saturn's North Polar hexagon}

- $\underline{\text { Masoud Rostami, (mrostami@lmd.ens.fr), Vladimir Zeitlin, }}$ Aymeric Spiga

This is the Authors' Original Manuscript (AOM); that is, the manuscript in its original form; a "preprint".

Published online in the ICARUS: 19 June 2017

https://doi.org/10.1016/j.icarus.2017.06.006

DOI: $10.1016 /$ j.icarus.2017.06.006 


\title{
On the dynamical nature of Saturn's North Polar hexagon.
}

\author{
Masoud Rostami ${ }^{a}$, Vladimir Zeitlin $^{b \star}$, and Aymeric Spiga ${ }^{c}$ \\ ${ }^{a, b, c}$ Laboratoire de Météorologie Dynamique (LMD)/IPSL, Université Pierre et \\ Marie Curie (UPMC), Paris, France \\ ${ }^{a, b}$ Ecole Normale Supérieure(ENS), Paris, France
}

\begin{abstract}
An explanation of long-lived Saturn's North Polar hexagonal circumpolar jet in terms of instability of the coupled system polar vortex - circumpolar jet is proposed in the framework of the rotating shallow water model, where scarcely known vertical structure of the Saturn's atmosphere is averaged out.

The absence of a hexagonal structure at Saturn's South Pole is explained similarly. By using the latest state-of-the-art observed winds in Saturn's polar regions a detailed linear stability analysis of the circumpolar jet is performed (i) excluding ("jet-only" configuration), and (2) including ("jet+vortex" configuration) the north polar vortex in the system. A domain of parameters: latitude of the circumpolar jet and curvature of its azimuthal velocity profile, where the most unstable mode of the system has azimuthal wavenumber 6 , is identified. Fully nonlinear simulations are then performed, initialized either with the most unstable mode of small amplitude, or with the random combination of unstable modes. It is shown that developing barotropic instability of the "jet+vortex" system produces a long-living structure akin to the observed hexagon, which is not the case of the "jet-only" system, which was studied in this context in a number of papers in literature. The north polar vortex, thus, plays a decisive dynamical role. The influence of moist convection, which was recently suggested to be at the origin of Saturn's north polar vortex system in the literature, is investigated in the framework of the model and does not alter the conclusions.
\end{abstract}

Keywords: Saturn's Hexagon ; Barotropic Instability ; Rotating Shallow Water Model

\footnotetext{
* Corresponding author. Email: zeitlin@lmd.ens.fr Address: LMD-ENS, 24 Rue Lhomond, 75005 Paris, France

Preprint submitted to Icarus

https://doi.org/10.1016/j.icarus.2017.06.006 DOI: $10.1016 /$ j.icarus.2017.06.006
} 


\section{Introduction}

Visible imagery acquired during the Voyager 1 \& 2 fly-by of Saturn in 1980-1981 revealed the hexagonal cloud pattern of Saturn's north pole at about $77^{\circ} \mathrm{N}$ planetographic latitude (Godfrey, 1988). As followed from these early observations, the motion of the associated cloud structures suggested that the structure is related to a circumpolar jet-stream, but the hexagon pattern itself appeared to be stationary (i.e. very close to the rotation of Saturn's interior). The early diagnostics were confirmed first in the 1990s by Hubble Space Telescope observations (SanchezLavega et al., 1993), and then in the 2000s by the Cassini orbiter firstly in thermal infrared images (Baines et al., 2009), followed by visible imagery once Saturn's northern pole came out of the winter darkness. The high spatial resolution of the Cassini visible and infrared images permitted to evidence the presence of the north polar vortex (NPV), in addition to the hexagonal jet. The exceptional duration of the Cassini mission allowed Sánchez-Lavega et al. (2014) to conclude that the hexagon resists seasonal changes, before Antunano et al. (2015) compiled the repeated cloud-layer observations of the hexagon to obtain a complete profiling of the winds in this structure. Thus far, such hexagonal feature has not been observed at Saturn's South pole.

The persisting hexagonal pattern in polar projected maps demonstrates that a prominent wavenumber 6 perturbation shapes Saturn's circumpolar jet stream at latitude $\approx 77^{\circ} \mathrm{N}$. An early interpretation by Allison et al. (1990) was that the anticyclonic North Polar Spot (NPS) vortex (not to confuse with the polar vortex), visible at that time in the vicinity of the hexagon forced a stationary planetary (Rossby) wave which gave the polar jet its hexagonal shape, but the apparent disappearance of the NPS between 1995 and 2004 invalidated this scenario (see Sayanagi et al. (2016) for the history of the observations of the NPS and the hexagon). The nature and origin of the hexagon has been addressed since then both in laboratory experiments and numerical models. Polygonal structures are often reported in rotating flow experiments, although the wavenumber 6 configuration remained elusive (Vatistas et al., 1994; Marcus and Lee, 1998; Jansson et al., 2006; Bergmann et al., 2011). Aguilar et al. (2010) discussed more specifically the relevance of rotating tank experiments to modeling Saturn's polar jet and concluded that the hexagonal structure could be resulting from the barotropic instability of the jet, and that the wavenumber 6 prominence is conditioned by the intensity of the jet and bottom friction. They were first to notice a dependence 
of the azimuthal wavenumber of the most unstable mode on the deformation radius in their instability analysis in the framework of the quasi-geostrophic (QG) model. Using a general circulation model with a domain limited to Saturn's northern polar region, and a polar jet as initial condition, Morales-Juberias et al. (2011) reproduced the structures found by Aguilar et al. (2010) in laboratory experiments: the hexagonal shape resulting from a vortex street formed by developing barotropic instability of the jet, with cyclonic and anticyclonic vortices at poleward and equator-ward sides of the jet, respectively. Yet, the vortices forming the street were too large and too strong, with respect to observations, and this mechanism was giving rise to larger propagation speed than the observed one (Sánchez-Lavega et al., 2014). Morales-Juberias et al. (2015) proposed an alternative "meandering jet" model which matches the morphology (including sharp potential vorticity, hereafter PV, fronts) and phase speed of Saturn's hexagon, provided that an ad hoc vertical shear of the jet is introduced. The same authors concluded that deep jets evolve into vortex streets and shallow jets evolve into meanders, both being possibly at the origin of the absence of seasonal variability of the hexagonal jet. Although the meandering jet model of Morales-Juberias et al. (2015) offers thus far the best agreement with the characteristics of the observed Saturn's hexagon, the putative meridional temperature gradients accompanying the vertical shear of the (supposedly shallow) polar jet are yet to be confirmed.

Despite these developments, there is not enough convincing dynamical explanation of the existence and origin of Saturn's North polar hexagon, as well as the absence of its counterpart at Saturn's South pole. In the present paper we propose an alternative approach to the problem. Our analysis is performed in the framework of a simple rotating shallow water (RSW) model, which is of use for modeling atmospheres of giant planets, cf. (Dowling, 1995). A specificity of our approach is that the model is considered in the polar tangent plane approximation, the so-called gamma-plane, in order to take into account the variations of the Coriolis parameter with latitude in the polar regions. The use of the model, which may be obtained by vertical averaging of the full primitive equations, is justified by the fact that information on the vertical structure of Saturn's atmosphere is scarce. All vertical structure being averaged out, the model is barotropic, in the sense that it does not contain any vertical shear, although it does allow for vertical stretching of fluid columns. In the same sense the instabilities in the model will be called barotropic below. The model combines simplicity with dynamical consistency and, with the astronomical parameters of Saturn being given, contains a single adjustable parameter: the effective Rossby deformation radius, $R_{d}$. The QG model used for theoretical analysis in Aguilar et al. (2010) can be obtained from RSW in the limit of vanishing Rossby numbers. The RSW model, which allows for efficient high-resolution numerical implementations, has been used for the analysis of various features of the Saturn's atmosphere: mid-latitude jets (Showman, 2007), 
equatorial super-rotating jet (Scott and Polvani, 2008), NPV (O'Neill et al., 2016) (in two-layer version with a moist-convective forcing in the latter work). Yet, to our knowledge, there are no studies in the literature applying the RSW model to the developing barotropic instability of the circumpolar Saturn's jet. The particularity of our approach is that we explore the dynamics in two different configurations of the mean zonal velocity profile: (i) considering only the $77^{\circ} \mathrm{N}$ circumpolar jet, with observed velocity profile, and no central vortex at $90^{\circ} \mathrm{N}$ (i.e. the traditional "jet-only" configuration considered in all above-cited papers), and (ii) considering the full "jet + vortex" system with the observed zonal velocity profile, which was not considered in the existing literature in this context. The state-of-the-art mean zonal velocity profiles on Saturn (Antunano et al., 2015) are used in linear stability studies and for initializations of nonlinear simulations.

The paper is organized as follows: in section 2 we introduce the model, discuss its general features and vortex solutions, present the analytical fits of the observed velocity profiles, and formulate the linear stability problem. Section 3 is devoted to the study of the jet-only configuration, with presentation of the results of the linear stability analysis and nonlinear simulations of the saturation of the instability. In section 4 we analyse the jet+vortex configuration along the same lines. Finally, in section 5 we present our conclusions and discussion. A discussion of the influence of the effects of moist convection upon the evolution of the instability is given in the Appendix.

\section{Rotating shallow water model for large-scale atmospheric motions, vortex solutions and their (in)stability}

\subsection{The model}

Rotating shallow water equations, in the absence of forcing and dissipation read:

$$
\begin{gathered}
\frac{D \boldsymbol{v}}{D t}+\left(f+\frac{v}{r}\right)(\hat{z} \times \boldsymbol{v})=-g \nabla h, \\
\frac{D h}{D t}+h \nabla \cdot \boldsymbol{v}=0 .
\end{gathered}
$$

Here, by anticipating the application to polar jets and vortices, we use the cylindrical coordinates on the plane $(r, \theta)$, and $\hat{z}$ is the unit vector normal to the plane. $\boldsymbol{v}=u \hat{r}+v \hat{\theta}$ is the horizontal velocity with $u, v$ denoting radial and azimuthal 
components, respectively, $D / D t=\partial / \partial t+\boldsymbol{v} \cdot \nabla$ denotes the material derivative, $\nabla=\hat{r} \partial_{r}+\hat{\theta}(1 / r) \partial_{\theta}$ is the gradient in the plane, where $\partial_{r}$ and $\partial_{\theta}$ denote partial derivatives with respect to corresponding arguments. $h$ is the thickness of the layer, $g$ is gravity and $f$ is the Coriolis parameter.

Bottom topography $b(r, \theta)$ can be easily introduced in the model by replacing $h$ by $h-b$ in (2.2), and can be used to parameterize the deep layers of the atmosphere. This would however introduce ad hoc parameters, which we want to avoid.

The RSW equations possess a Lagrangian invariant, the potential vorticity (PV):

$$
q=\frac{\hat{z} \cdot(\nabla \times \boldsymbol{v})+f}{h},
$$

where $\hat{z} \cdot(\nabla \times \boldsymbol{v})$ is relative vorticity. The PV anomaly

$$
P V A=q-f_{0} / H_{0}
$$

where $f_{0}$ and $H_{0}$ are reference values of Coriolis parameter and thickness of the layer (see below), will be used for diagnostics in what follows. We consider the RSW equations in the polar tangent plane, the so-called $\gamma$-plane, where the effects of sphericity in the Coriolis parameter are taken into account in the lowest-order approximation:

$$
f=f_{0} \sin (\phi)=f_{0}\left(1-\frac{1}{2 R_{s}{ }^{2}} r^{2}+\ldots\right)=f_{0}\left(1-\gamma^{*} r^{* 2}+\ldots\right) ; \quad 0 \leq r^{*} \leq \frac{R_{s}}{R_{d}} .
$$

Here $f_{0}=2 \Omega_{s}, \phi$ is planetographic latitude, $\Omega_{s}$ and $R_{s}$ represent Saturn's angular velocity and radius, respectively (we will neglect the effects of non-sphericity in what follows). Approximate values for the mean Saturn radius and $f_{0}$ are respectively $55000 \mathrm{~km}$ and $3.2 \times 10^{-4} \mathrm{~s}^{-1}$ (Read et al., 2009b; Sánchez-Lavega et al., 2014). As is well-known, the rotating shallow water system possesses an internal scale, the Rossby deformation radius $R_{d}=\sqrt{g H_{0}} / f_{0}$.

Equations (2.1), (2.2) can be obtained by vertical averaging between two material surfaces of the full three-dimensional primitive equations for the atmosphere in pseudo-height isobaric coordinates, e.g. (Bouchut et al., 2009), when one of these surfaces is considered fixed (a constant pressure level), and one is free. The potential temperature is considered uniform through the layer in this approximation, although an extension of the model including horizontal potential temperature gradients is possible. In this case $g$ is the gravity acceleration of Saturn, and $H_{0}$ is the thickness of the layer. The model can be also considered as a two-layer model with strong disparity in depths and/or densities of the layers. In this case $H_{0}$ plays 
a role of equivalent depth, and $g$ is so-called reduced gravity, i.e. gravity weighted with the stratification parameter. The deformation radius in this interpretation is related to stratification properties of the atmosphere. Whatever the interpretation is, at a given rotation rate $f_{0} / 2$, the deformation radius $R_{d}$ is the only free parameter of the model, as the velocity scale can be taken to be $\sqrt{g H_{0}}$.

In terms of velocity components, the momentum and mass conservation equations of the model read:

$$
\begin{gathered}
\frac{D u}{D t}-\frac{v^{2}}{r}-f v=-g \partial_{r} h, \\
\frac{D v}{D t}+\frac{u v}{r}+f u=-g \partial_{\theta} h, \\
\partial_{t} h+\frac{1}{r} \partial_{r}(h r u)+\frac{1}{r} \partial_{\theta}(h v)=0 .
\end{gathered}
$$

\subsection{Vortex solutions}

Stationary axisymmetric vortex solutions of the equations (2.6) - (2.8) are those with zero radial velocity $u=0$, some axisymmetric distribution of azimuthal velocity $v=V(r)$, and the thickness field $H(r)$ related to $V(r)$ through the cyclogeostrophic (gradient wind) balance:

$$
\frac{V^{2}}{r}+f V=g \partial_{r} H
$$

For a given $V(r)$, corresponding thickness profile $H(r)$ can be obtained by integration of equation (2.9). Note that in the QG model used for theoretical analysis in Aguilar et al. (2010) the centrifugal acceleration (the first term in (2.9)) is neglected. While this approximation is fully justified for the circumpolar jet, it is less so for the central vortex. Herein we consider solutions of (2.9) with $V(r)$ corresponding to the observed time- and zonally averaged profiles of Saturn's zonal wind (Antunano et al., 2015). These profiles, with error margins, are represented by dashed lines in Figure 1.

It is useful to have a simple analytic expression mimicking the observed velocity profiles. Although the linear stability analysis can be accomplished directly with digitized observational velocity profile, it is convenient to control the shape of the velocity profile in terms of a small number of parameters. Dependence on 

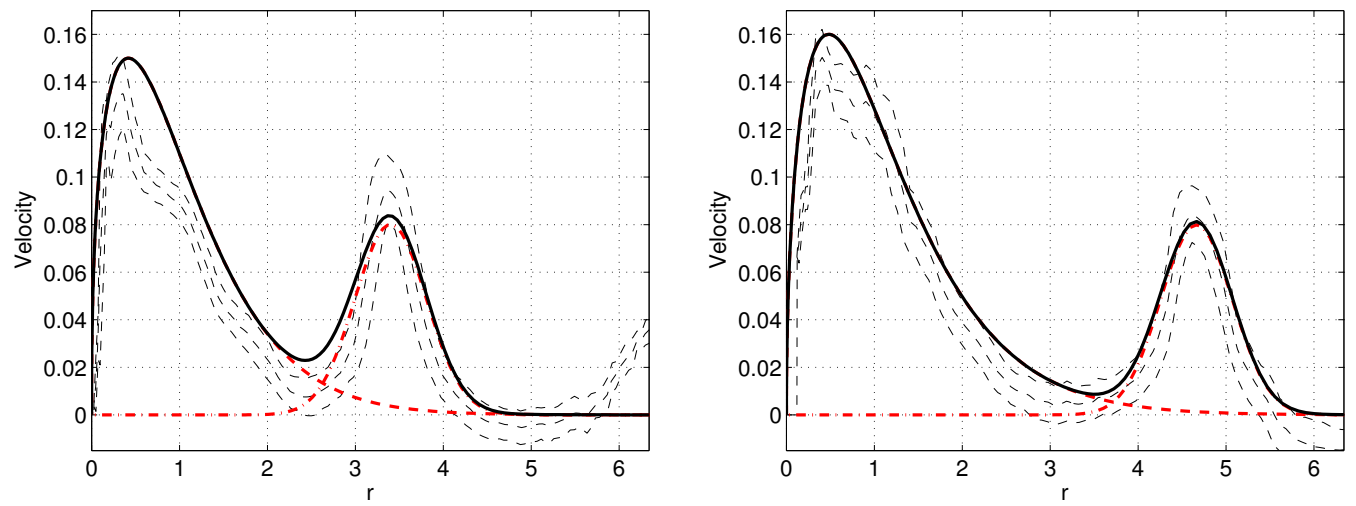

Figure 1. Observed zonal velocity profiles in the northern (left panel) and southern (right panel) hemispheres and their fits used in the paper. Three dashed lines represent the observed non-dimensional mean and error margins of the averaged zonal velocity (Antunano et al., 2015), and the solid line is the composite of two model profiles (in red) fitting the $N P V$ and the circumpolar jet. The fit of the velocity profile of the $N P V$ gives $\epsilon=0.15, \alpha=0.42, \beta=1.3, r_{0}=0, m=1$ and that of the circumpolar jet gives $\epsilon=0.08, \alpha=0, \beta=2, r_{0}=3.37, m=3$. Similarly, south polar vortex correspond to $\epsilon=0.16, \alpha=0.5, \beta=1.2, r_{0}=0, m=1$ and southern circumpolar jet jet to $\epsilon=0.08, \alpha=0, \beta=2, r_{0}=4.6, m=3 . R_{d} \approx 3200 \mathrm{~km}$ is taken for both poles.
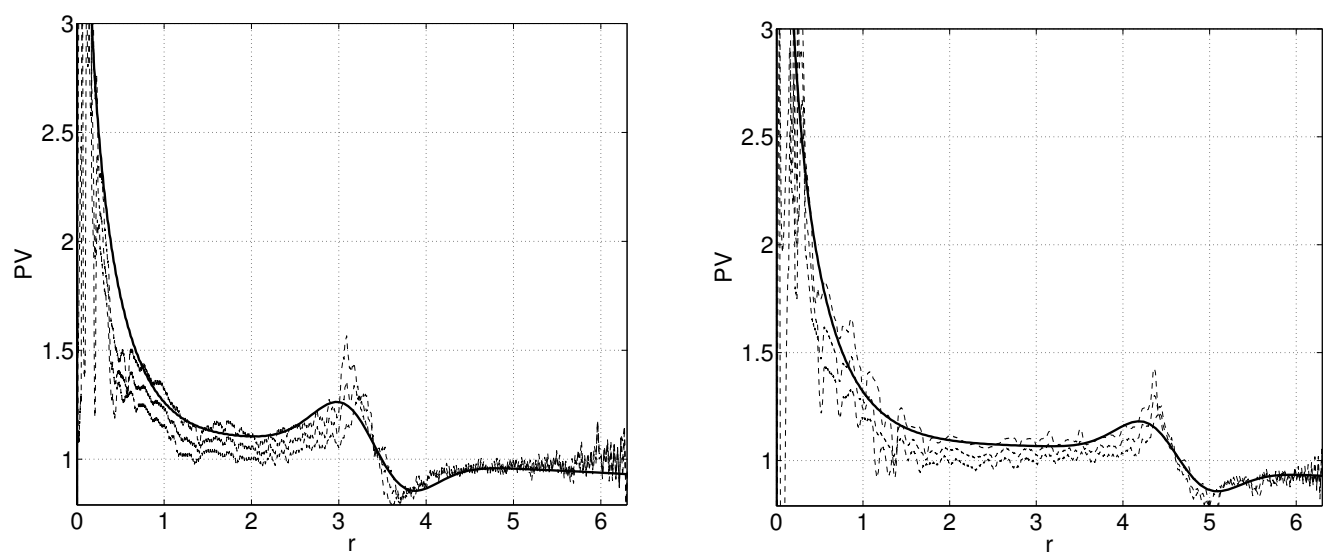

Figure 2. $P V$ distributions corresponding to observed velocity profiles of Fig. 1 in the northern (left panel) and southern (right panel) hemispheres and their analytic fits.

these parameters could be then analysed. The PV, which is a key characteristic of the flow, is very noisy, if determined directly from the observed profiles, cf. Fig. 2, whereas a PV field computed from the analytic fit enables a straightforward analysis of possible instability, see below. For initialization of the non-linear RSW simulations, the use of the analytic fit allows for straightforward integration of (2.9), and thus diminish the discretisation errors. Therefore, we fit the peaks of 
the velocity distribution with the help of the following simple formula:

$$
V(r)=\epsilon\left(r-r_{0}\right)^{\alpha} e^{-m\left(r-r_{0}\right)^{\beta}}, \quad \alpha, \beta, \epsilon, m \geq 0
$$

where $\epsilon$ measures the intensity of the velocity field, $r_{0}$ tunes the distance of the velocity peak of the jet from the pole, and other parameters allow to fit the shape of the distribution ( $r_{0}=0$ for the central vortex and $\alpha=0$ for the jet). All velocity profiles are normalized by their maximum values, so the maximum value of velocity is equal to $\epsilon$. By applying this formula to both central vortex and circumpolar jet (red curves in Fig. 1), we get synthetic profiles represented by solid black lines in the figure. We do not seek to reproduce the "shoulder" in the velocity profile of the central vortex, as it turns out that it is inessential for the dominant long-wave instability of the system which we are interested in (a test study was performed to check this fact).

The stability of any velocity profile $V(r)$ (and corresponding $H(r)$ obtained from the cyclo-geostrophic balance) with respect to small perturbations can be analyzed by standard means. We represent each field as the solution in question and a small perturbation (denoted by a prime):

$$
\begin{gathered}
u(r, \theta, t)=u^{\prime}(r, \theta, t), \\
v(r, \theta, t)=V(r)+v^{\prime}(r, \theta, t), \\
h(r, \theta, t)=H(r)+h^{\prime}(r, \theta, t),
\end{gathered}
$$

and inject these expressions in the full system (2.6) - (2.8). Retaining only the linear contributions in perturbation terms, we are looking for normal-mode solutions with harmonic dependence on time and polar angle

$$
\left(u^{\prime}, v^{\prime}, h^{\prime}\right)(r, \theta, t)=\operatorname{Re}\left[(i \tilde{u}, \tilde{v}, \tilde{h})(r) e^{i(l \theta-\omega t)}\right]
$$

where $l$ and $\omega$ are the azimuthal wavenumber and the complex eigenfrequency, $\omega=\omega_{R}+i \omega_{I}$. We thus formulate the problem as an eigenvalue problem for eigenfrequencies $\omega$. A positive imaginary part for an eigenfrequency $\omega_{I}$ corresponds to an instability with a linear growth rate $\sigma=\omega_{I}$.

Following common practice, it is convenient to formulate this eigenproblem in nondimensional terms. By using the deformation radius $R_{d}$ as the scale for $r, f_{0}^{-1}$ as the scale for $t$, and $\sqrt{g H_{0}}$ as the scale for velocity, the eigenproblem in question 
takes the following form:

$$
\left[\begin{array}{ccc}
\frac{l V^{*}}{r^{*}} & \left(f^{*}+\frac{2 V^{*}}{r^{*}}\right) & -D_{r^{*}} \\
\left(f^{*}+\frac{V^{*}}{r^{*}}+D_{r^{*}} V^{*}\right) & \frac{l V^{*}}{r^{*}} & \frac{l}{r^{*}} \\
H^{*} D_{r^{*}}+\frac{1}{r^{*}} D_{r^{*}}\left(r^{*} H^{*}\right) & \frac{l H^{*}}{r^{*}} & \frac{l V^{*}}{r^{*}}
\end{array}\right] \times\left[\begin{array}{c}
\tilde{u} \\
\tilde{v} \\
\tilde{\eta}
\end{array}\right]=\omega\left[\begin{array}{c}
\tilde{u} \\
\tilde{v} \\
\tilde{\eta}
\end{array}\right]
$$

where variables marked with the asterisks are non-dimensional, and $D_{r^{*}}$ denotes the differentiation with respect to $r^{*}$. The non-dimensional $\gamma^{*}=O\left(10^{-3}\right)$. It should be noted that the parameter $\epsilon$ acquires in this way a meaning of the Rossby number.

Pseudospectral collocation method (Trefethen, 2000) is used for linear stability analysis of the eigen-problem (2.15). The system is discretized over $N$-point grid and $D_{r^{*}}$ becomes the Chebyshev differentiation operator. To avoid the Runge phenomenon, Chebyshev collocation points are used, following Lahaye and Zeitlin (2015) where the method of Boyd (1987) was adapted to the stability problem of circular vortices.

\section{Instability of the "jet-only" configuration and its nonlinear satura- tion}

In this section, we analyse the instability of the North Polar circumpolar jet in the absence of the central vortex ("jet-only" configuration), following the existing studies in the literature (Aguilar et al., 2010; Morales-Juberias et al., 2011, 2015), albeit in a different model. The velocity profile of the jet is fitted by a Gaussian, i.e. the profile (2.10) with $\alpha=0$. The non-dimensional amplitude of the jet is $\epsilon=0.08$. The values of parameters $m$ and $r_{0}$ corresponding to the most reasonable fit of the observations with error bars (see Fig. 1) are $m=3$ and $r_{0}=3.37$.

\subsection{Results of the linear stability analysis}

We present in this subsection the results of linear stability analysis for the "jetonly" configuration, carried out along the lines of section 2. The barotropic instability of zonal jets, as well as its nonlinear saturation, are well understood in geophysical fluid dynamics. In the framework of the RSW model sufficient conditions of stability of zonal jets are given by Ripa's criteria (Ripa, 1990). Like for the 
famous Rayleigh - Kuo stability criterion (which should be adapted, however, to the current $\gamma$ - plane context) the meaning of this criterion is that in order to have the instability the PV gradients should change sign within the flow. Physically, this means that there should be a possibility for Rossby waves, which owe their existence to PV gradients, to propagate in opposite directions in the flow, phaselock, and grow in amplitude. As follows from Fig. 2 there is indeed a change of sign of the gradient of PV, which is by the way more pronounced in the Northern hemisphere, and hence we expect the barotropic instability. The main question then is: under what precise circumstances the mode with azimuthal wavenumber $l=6$ is the dominant instability mode of the circumpolar jet? Our linear stability analysis demonstrates that for the location of the jet at the distance from the pole $r_{0}$ corresponding to the observations, the dominant instability mode has an azimuthal wavenumber $l=6$ for a choice of $R_{d} \approx 3200 \mathrm{~km}$. This value of $R_{d}$ is in line with the values of $2500-3000 \mathrm{~km}$ adopted in Aguilar et al. (2010) and O'Neill et al. (2016) (cf. their appendix 2), and are of the same order of magnitude as the estimates from observations in Read et al. (2009a), although about twice larger. Yet $R_{d}$ remains to be better constrained by observations.

Changing the radial (latitudinal) position of the jet, $r_{0}$, changes the wavenumber of the most unstable mode. This is shown in Fig.3, where the growth rates of unstable modes as a function of $\epsilon$ and $r_{0}$ are displayed in a range of values of $\epsilon$ and $r_{0}$ covered by our linear stability analysis). Fig. 3 shows that the azimuthal wavenumber of the most unstable mode varies from $l=2$ to $l=8$ with $r_{0}$ increasing from 2 to 5 in non-dimensional terms. For some values of $r_{0}$, several modes exhibit very close growth rates, especially at small $\epsilon$ : in such configuration a combination of these modes would eventually shape a circumpolar jet without pronounced polygonal pattern. It is worth noting that an increase of velocity amplitude of the jet, $\epsilon$, does not change the azimuthal wavenumber of the most unstable mode, but increases the growth rate, cf. Fig.3.

It should be emphasized, however, that even a small difference in growth rates yields exponentially-growing differences between corresponding wave patterns, whence the primary importance of the leading mode.

In Figure 4 we present the regions in the parameter plane $r_{0}-m$ where unstable modes with a given $l$ have the highest growth rate at fixed $R_{d}$. Higher values of $m$ correspond to stronger curvatures of the velocity distribution. The value of $R_{d}$ also influences the results. For larger values of $R_{d}$, the observed position of the maximum jet velocity shifts closer to the pole in non-dimensional terms and, as follows from the results described in the previous paragraph, the azimuthal wavenumber of the leading instability becomes smaller, and vice-versa. By reversing the argument, 

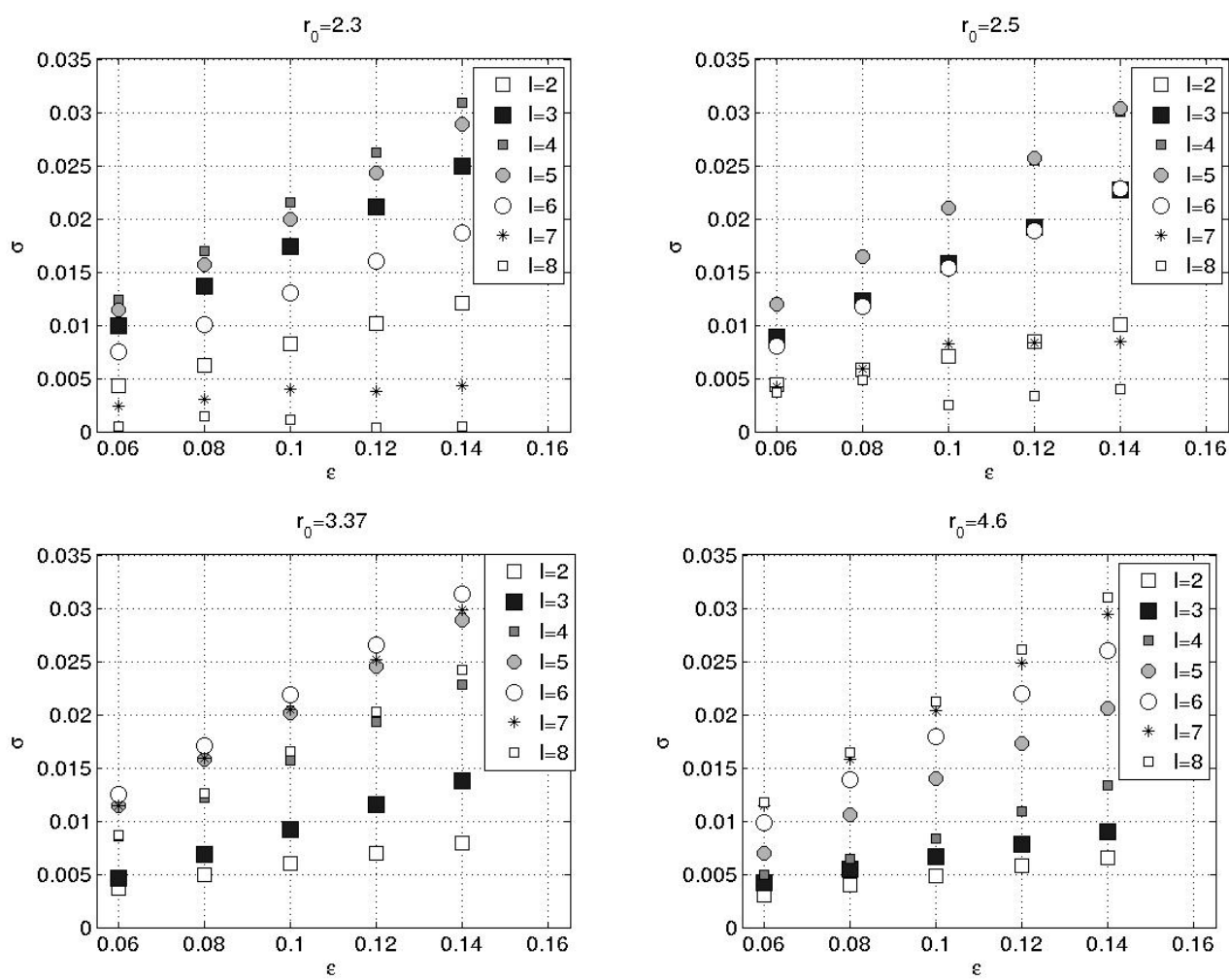

Figure 3. Dependence of the growth rate of the unstable modes on azimuthal wavenumber for different latitudinal positions of the jet. $V(r)$ is Gaussian, with $m=3, \alpha=0, \beta=2, R_{d} \approx 3200 \mathrm{~km}$.

from our analysis and from the fact that the observed dominant wavenumber is $l=6$, we can infer an estimate for $R_{d} \approx 3200 \mathrm{~km}$.

An important unsolved problem is the difference in morphology between the hexagonal northern polar jet and the southern polar jet which does not exhibit a pronounced polygonal form in Saturn's atmosphere, as follows from the observations (Antunano et al., 2015). Our linear stability analysis of "jet-only" configuration provides a clue for explanation. As follows from Fig. 1, at a given value of $R_{d}$, the value of $r_{0}$ is higher in the southern hemisphere (i.e. the southern jet is farther from the pole), which yields a leading instability with $l \geq 8$ (Fig. 3), and hence no hexagonal pattern at Saturn's South Pole, although a partial polygonal structure was discussed in the literature (Vasavada et al., 2006). 

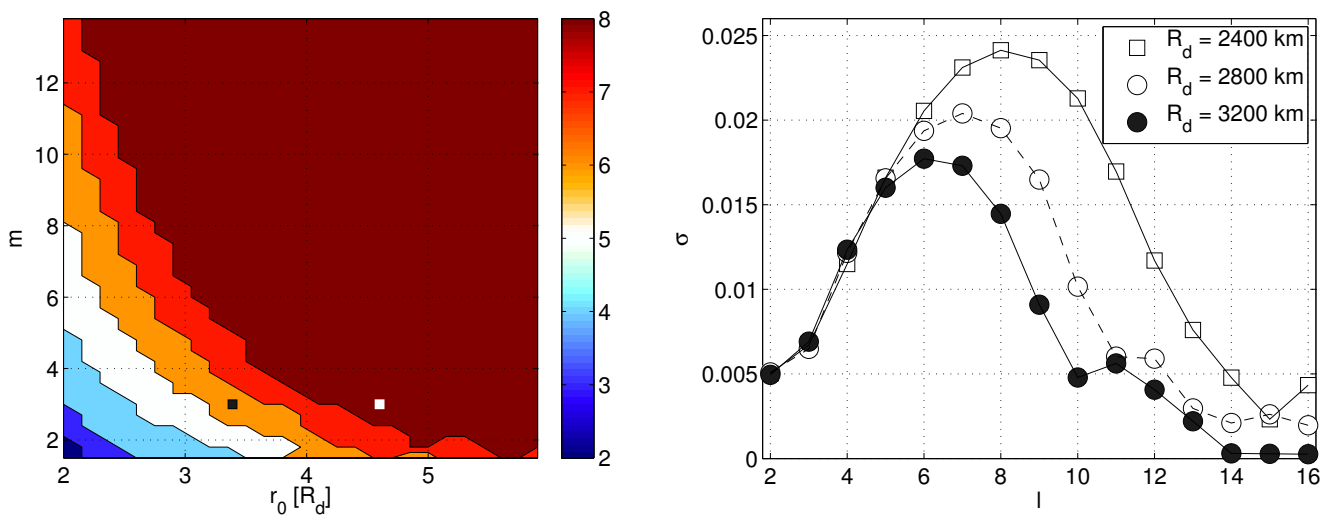

Figure 4. Left panel: Distribution of azimuthal wavenumbers of the most unstable modes in the plane of parameters $r_{0}-m$. Black (white) square corresponds to the observed profile in the northern (southern) hemisphere. $V(r)$ is Gaussian, with $\epsilon=0.08, \alpha=0, \beta=2$. Azimuthal wave-numbers larger than eight are not included. Right panel: Dependence of the growth rates of the unstable modes of the "jet-only" configuration in northern hemisphere of the value of equivalent depth. Variation of the growth rate with azimuthal wavenumber for the jet corresponding to the black square in the left panel corresponds to $R_{d} \approx 3200 \mathrm{~km}$.

\subsection{Non-linear saturation of the instability}

In order to study the nonlinear saturation of the instability identified in the previous subsection, we perform direct numerical simulations with a well-balanced entropy-satisfying finite-volume scheme (Bouchut, 2007) for the RSW equations. The numerical scheme was successfully tested in studies of the saturation of the barotropic instability of jets and vortices (Lambaerts et al., 2011; Lahaye and Zeitlin, 2015). The scheme has no explicit dissipation, only a numerical one. The dissipation is concentrated in the zones of strong gradients of velocity and thickness. For flows with low Rossby numbers, as is the case here, the scheme is practically dissipationless and allows for high-resolution long-time simulations. The simulations below are initialized with the most unstable mode of small amplitude (several per cent with respect to the background flow) superimposed onto the jet, or with an ensemble of unstable modes with random phases.

The evolution of the instability follows the well-known scenario of the saturation of the barotropic instability. Namely, a series of vortices of opposite sign appear at the crests and lows of the initial unstable wave, cf. Fig.5. This is similar to the vortex-street patterns observed in simulations of Morales-Juberias et al. (2011) and laboratory experiments of Aguilar et al. (2010). As time goes on, the vortices intensify, resulting in a strong deformation of the polar jet. Although the 

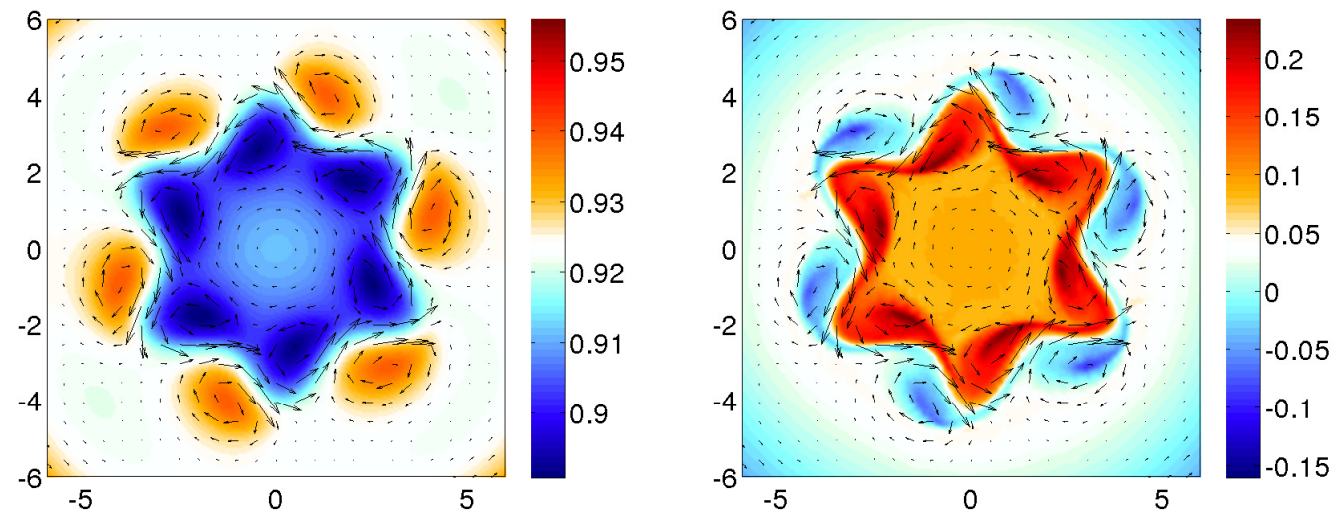

Figure 5. Snapshots of the pressure (left panel) and potential vorticity anomaly (right panel) at $t=150 f_{0}^{-1}$ during the saturation of the instability in the "jet-only" configuration.

outcome does preserve a six-fold symmetry of the initial unstable mode, the observed hexagon with quasi-straight sides (corresponding to sharp PV gradients) is not reproduced. Moreover, as follows from Fig. 6, the azimuthally averaged velocity of the resulting structure displays formation of counter-jets at both sides of the original jet, which is typical for the saturation of the barotropic instability (e.g. (Lambaerts et al., 2011)), and does not match the observations. At later stages the hexagonal structure practically disappears (cf Fig. 7). The evolution of radial distribution of zonally averaged PVA, as follows from the simulations, is presented in Fig. 6. As follows from the figure, the flow reorganizes by diminishing PV gradients and flattening the radial PV distribution in the region between the circumpolar jet and the NPV , and thus tending to "cure" the instability, but this process is not yet over at $t=450 f_{0}^{-1}$. Indeed, opposite PV gradients of comparable strength are still present in the flow at this stage, thus maintaining the barotropic instability. We do not trace further the process of saturation which is well-known and leads to complete "healing" of the instability, appearance of counter-jets (although here we have a new element with respect to classical studies element, the $\gamma$ - effect), and does not produce a quasi-stationary hexagonal structure. Note that the (diminishing) maximum of PVA shifts poleward during the saturation of the instability.

Although the $l=6$ instability is dominant, the growth rates of $l=5$ and $l=7$ modes are rather close to the growth rate of $l=6$ (Fig. 3, left bottom panel). If the initialization of direct numerical simulations is made with a combination of these modes with equal amplitudes and random phases, the velocity distribution is still meandering and possesses zonal counter-jets at the intermediate stages, and does not exhibit the hexagonal shape at late stages (not shown). 

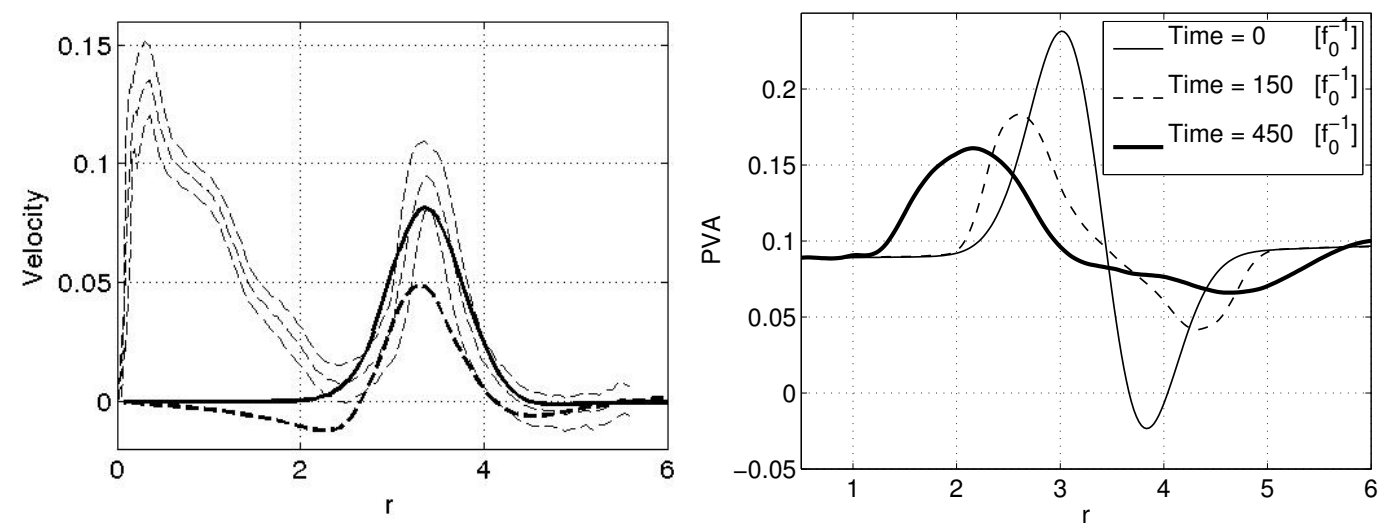

Figure 6. Left panel: Superposition of the observed mean azimuthal velocity profile with error margins (thin dashed), model azimuthal velocity profile (solid), and mean azimuthal velocity profile (dashed) at $t=150 f_{0}{ }^{-1}$ during the saturation of the instability in the "jet-only" configuration. Right panel: Evolution of the radial distribution of zonally averaged PVA in the "jet-only" configuration.
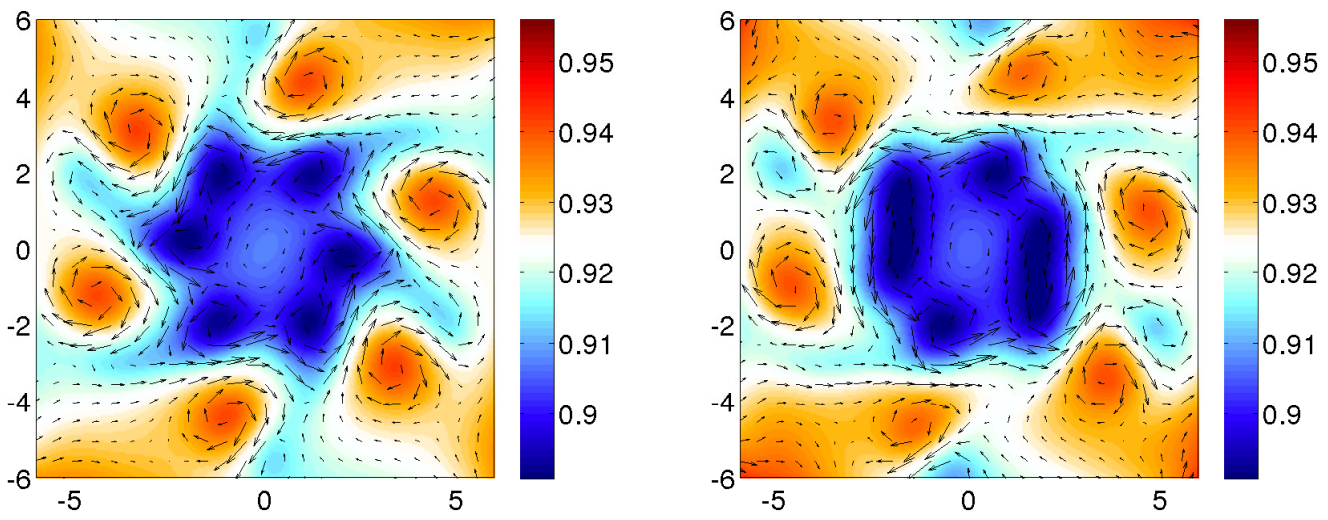

Figure 7. Same as in the left panel of Fig 5, but for $t=350 f_{0}^{-1}$, (left panel), and $450 f_{0}^{-1}$ (right panel).

\section{Instability of the "jet + vortex" configuration and its nonlinear sat- uration}

It should be stressed that the "jet-only" approximation of section 3, which is being overwhelmingly used in the literature thus far, would be valid if the jet and the vortex were spatially well separated. As follows from Fig. 1, the velocity peaks are separated by approximately $3 R_{d}$. A well-known property of the RSW is that the deformation radius $R_{d}$ plays a role of a "screening" radius beyond which the velocity field generated by a vortex falls off exponentially. In spite of their rather well-separated velocity peaks, the velocity distributions of the central vortex and of the circumpolar jet are not sufficiently far from each other to prevent 

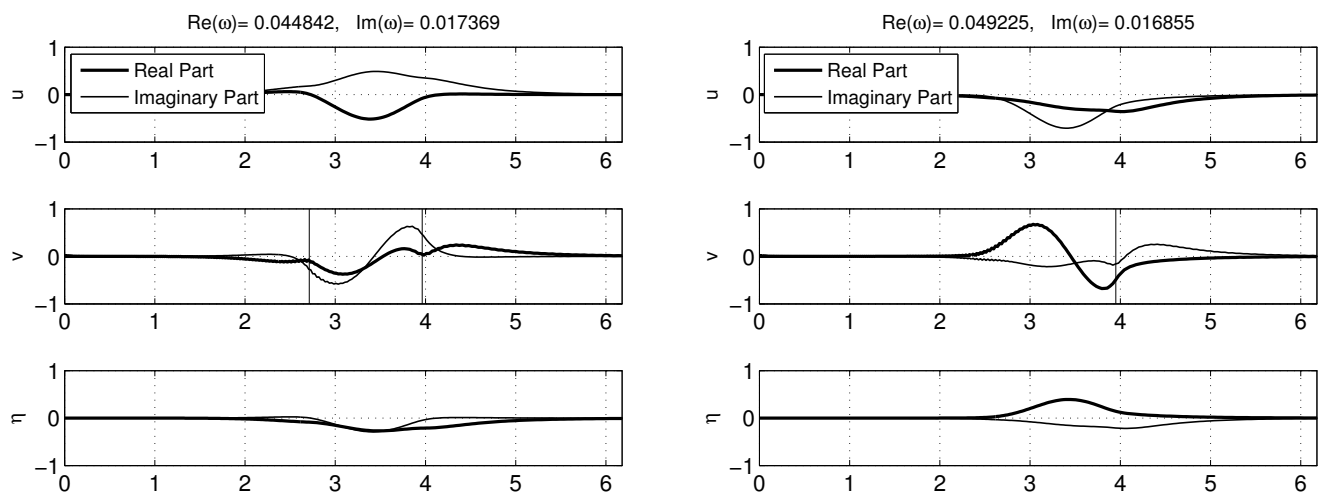

Figure 8. Structure of the most unstable mode with $l=6$ of "jet-only" configuration (left panel) and of "jet+vortex" one (right panel)in the northern hemisphere corresponding to the background velocity profile of Fig.1. Vertical lines represent positions of critical levels.

the central vortex influencing the perturbations of the jet. We will show below that this influence changes the evolution of the instability, although the most unstable mode of the composite jet + vortex system remains practically the same as in the "jet-only" case.

\subsection{Results of the linear stability analysis}

The most important result following from the linear stability analysis in the "jet+vortex" configuration (Fig. 8) is that the most unstable mode has $l=6$ as in the "jet-only" case, and that its structure is practically the same. The big picture of the dependence of the azimuthal wavenumber of the most unstable mode on the parameter $r_{0}$ is also close to what was observed in the "jet-only" configuration, as follows from Fig. 10. The configuration corresponding to the South Pole has the most unstable mode with $l=8$ (at the value $R_{d} \approx 3200 \mathrm{~km}$ used as a reference for comparison with the "jet-only" case). The position of the southern polar jet being much farther from the pole in comparison with the northern polar jet, we can expect, as was the case in the "jet-only" configuration, that instability happens at higher $l$ than the $l=6$ leading to the hexagonal structure. The most unstable $l$ can be pushed even higher by diminishing $R_{d}$, as follows from Fig. 11 . The configuration corresponding to the South Pole has the most unstable mode with $l=8$ (at the value $R_{d} \approx 3200 \mathrm{~km}$ used as a reference for comparison with the "jet-only" case). The position of the southern polar jet being much farther from the pole in comparison with the northern polar jet, we can expect, as was the case in the "jet-only" configuration, that instability happens at higher $l$ than the $l=6$ 

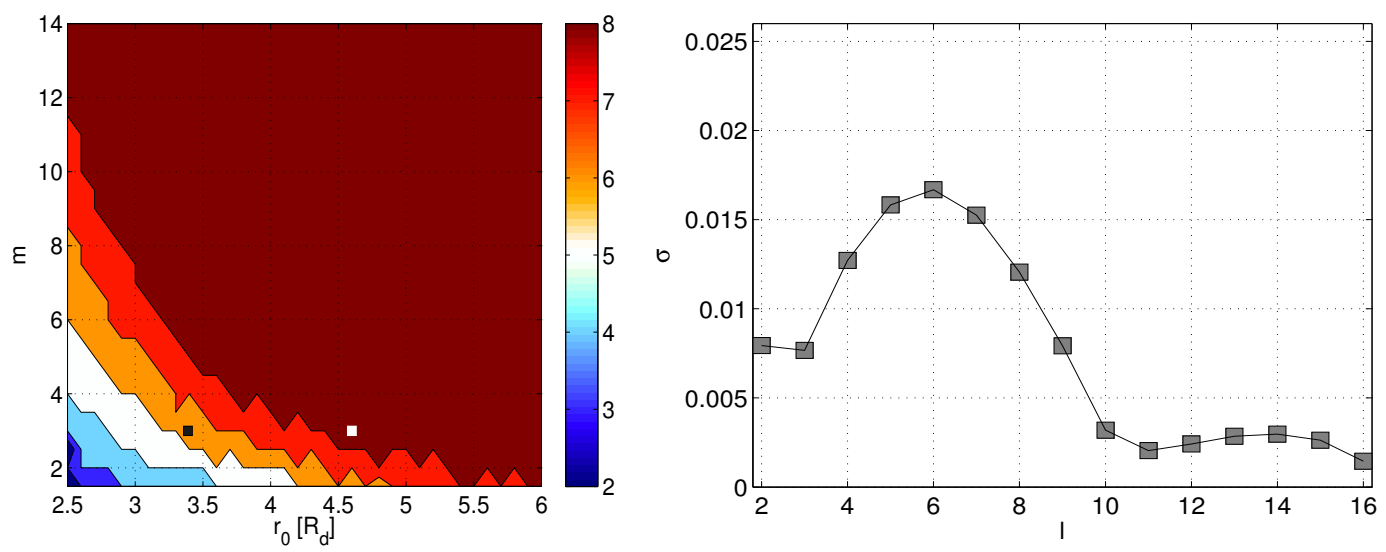

Figure 9. Same as in Fig.4, but for the "jet+vortex" configuration. The white square corresponds to the parameters in the right panel of Fig. 1.
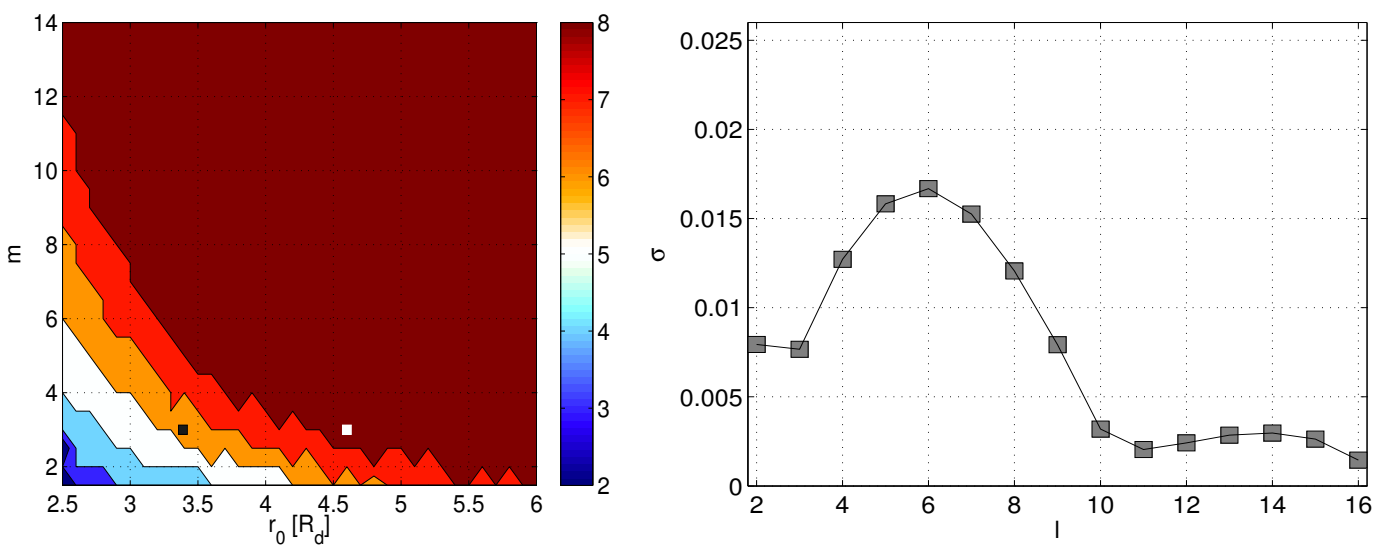

Figure 10. Same as in Fig.4, but for the "jet+vortex" configuration. The white square corresponds to the parameters in the right panel of Fig. 1.

leading to the hexagonal structure. The most unstable $l$ can be pushed even higher by diminishing $R_{d}$, as follows from Fig. 11. As one can infer from the observations (Antunano et al., 2015) the southern circumpolar jet has a much higher azimuthal wavenumber than $l=8(l=10-11)$, we thus may conclude that the equivalent depth near the South Pole is, by some reason, smaller than in the vicinity of the North Pole.

\subsection{Non-linear saturation of the instability}

Direct numerical simulations of the evolution of the instability of the "jet+vortex" system were performed using the same procedure as in the "jet-only" case. We 

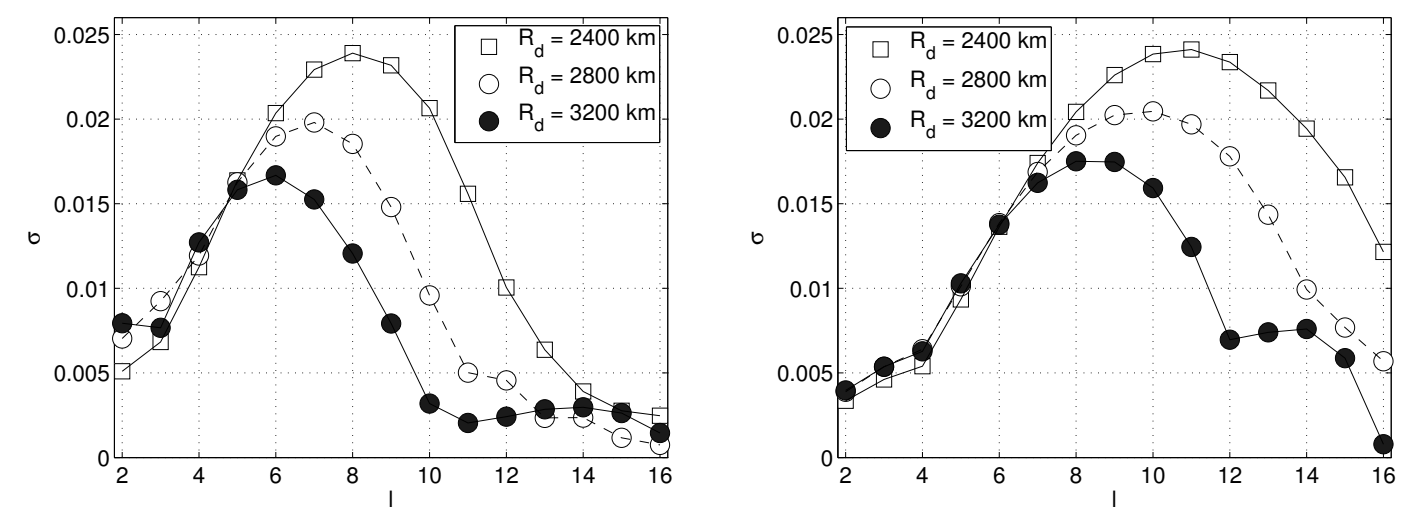

Figure 11. Linear growth rates $\sigma$ of different azimuthal modes of "jet+vortex" configuration at the North Pole (left panel) and South Pole (right panel) at different values for $R_{d}$. The non-dimensional velocity profiles are the same as Fig.1.

present in Fig. 12 the snapshots of pressure and potential vorticity anomaly at times $t=150 f_{0}^{-1}$ and $t=1000 f_{0}^{-1}$ clearly showing the formation and maintenance of a hexagonal structure matching the observed feature. Compared with the evolution of the "jet-only" configuration in Figs. 5, 7 the "jet+vortex" case exhibits much less deformation of the jet with time, resulting in the long-living quasistationary hexagonal pattern exhibiting sharp straight PV boundaries, which was not the case in the "jet-only" configuration. The evolution of the zonally averaged PVA is presented in Fig. 13. As follows from the figure, the jet+vortex configuration "cures" the instability at $t=1000 f_{0}^{-1}$. Positive PV gradients become small and are shifted farther from the zone of negative PV gradients of the jet. This prevents effective coupling of Rossby waves moving in opposite direction and, thus, eliminates the cause of instability. We performed a linear stability analysis of the mean zonal velocity profile corresponding to the PVA profile at $t=1000 f_{0}^{-1}$ of Fig. 13 and found that although the unstable modes still exist, their growth rates are at least one order of magnitude less than those of unstable modes of initial profile. Hence, in accordance with qualitative considerations above, the instability does cure itself. This process, is, however, not sufficient to explain by itself why the hexagonal structure of finite amplitude persists.

The inclusion of the NPV thus completely changes the evolution of the circumpolar jet. This could be further demonstrated by comparing the evolution of the mean azimuthal velocity in the "jet+vortex" case presented in Fig. 14 with that of the "jet-only" case in Fig. 6. The results are presented for two different intensities of the central vortex: one corresponding to the observations, and another one of the same shape but of higher amplitude. In the second case the system reaches a quasi-equilibrium configuration close to the observed one in high latitudes. Yet, 

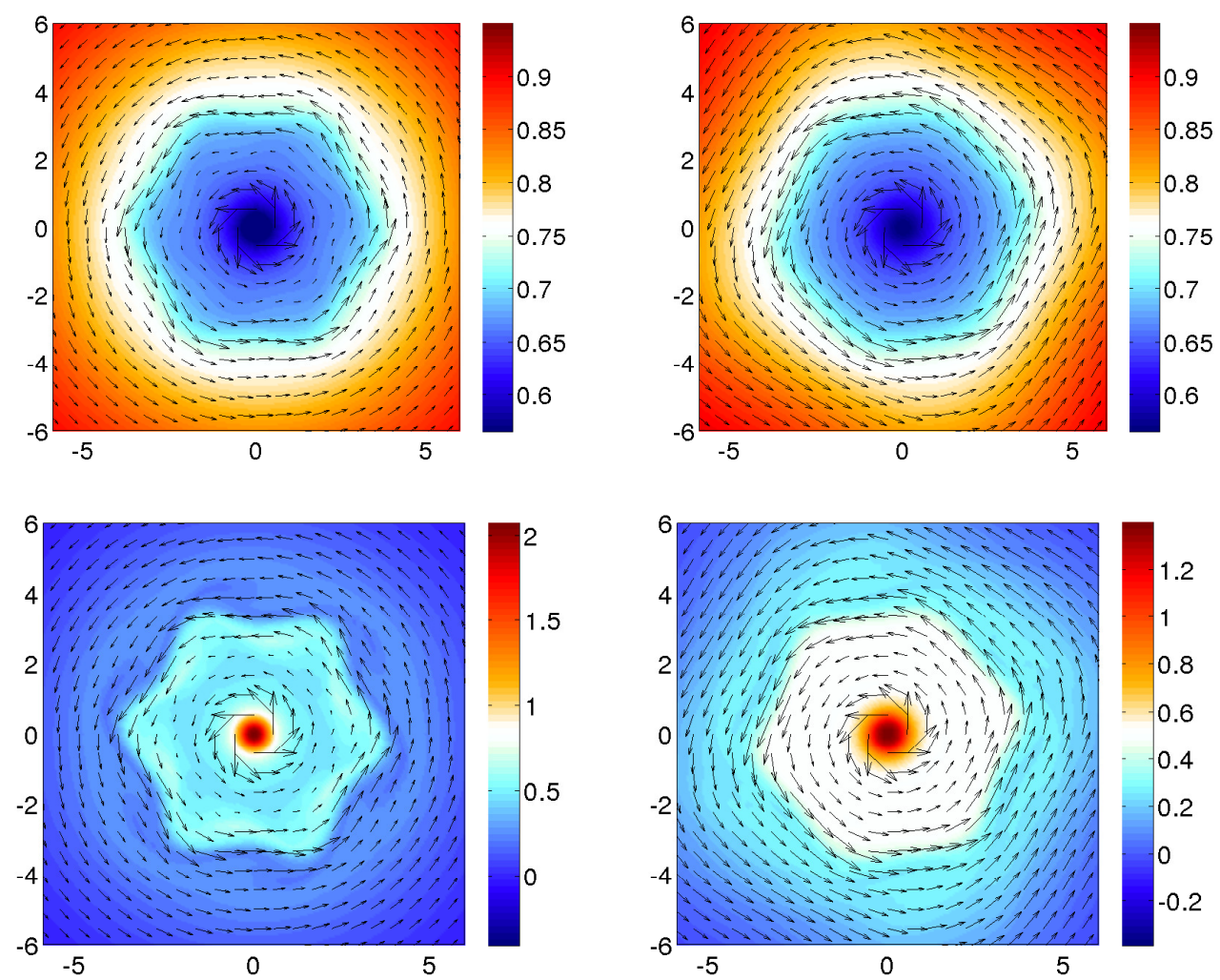

Figure 12. Upper row: Snapshots of pressure distribution in "jet+vortex" configuration at time $=150,1000 f_{0}{ }^{-1}$ respectively, left and right panels. Lower row: corresponding potential vorticity anomaly. $V(r)$ is the same as Fig.1. For better visibility the colorbars are not the same in two lower figures.

a discrepancy is observed in low latitudes, where the velocity field in simulations does not match observations. It should be stressed that our goal is, in the first place, to understand the dynamical role of the polar vortex, so we did not seek to represent the details of the velocity field beyond the jet with our simple fit. There are also technical reasons for this decision. First, the $\gamma$ plane approximation is losing its accuracy while moving farther from the pole, so trying to reproduce fine details of the velocity profile does not make much sense. Second, in order to prevent emitted inertia-gravity waves from returning back to the vortex, sponge boundary conditions were imposed at the boundary of the computational domain at $r \approx 10$. The mean velocity field, therefore should fall off to zero sufficiently far from the sponges, to be not perturbed by these latter. However, the (weak) retrograde jet which is present in the data could play a role. For example, an exchange of angular momentum between retrograde and prograde jets in Saturn's atmosphere was reported in Liu and Schneider (2015). Simulations on the whole sphere are needed in order to correctly reproduce this part of the velocity profile, 


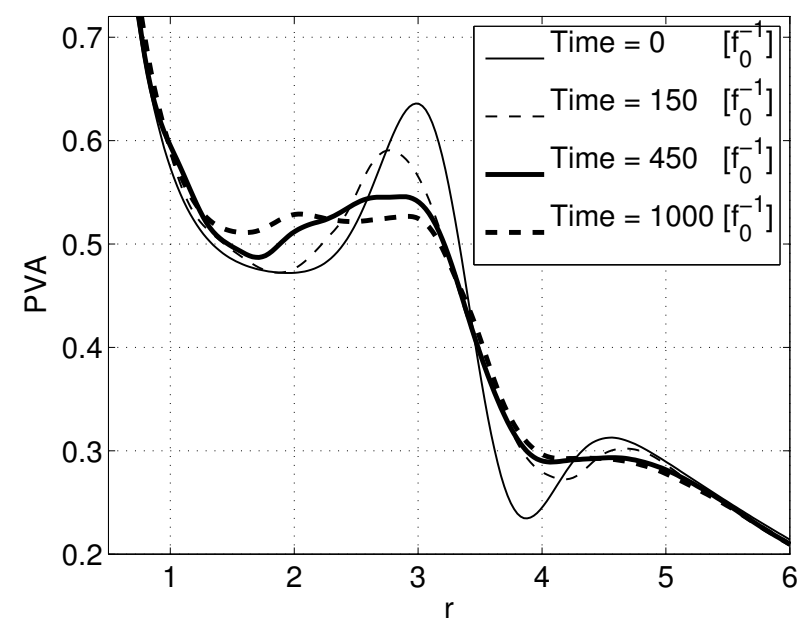

Figure 13. Evolution of the radial distribution of zonally averaged PVA in the "jet+vortex" configuration.
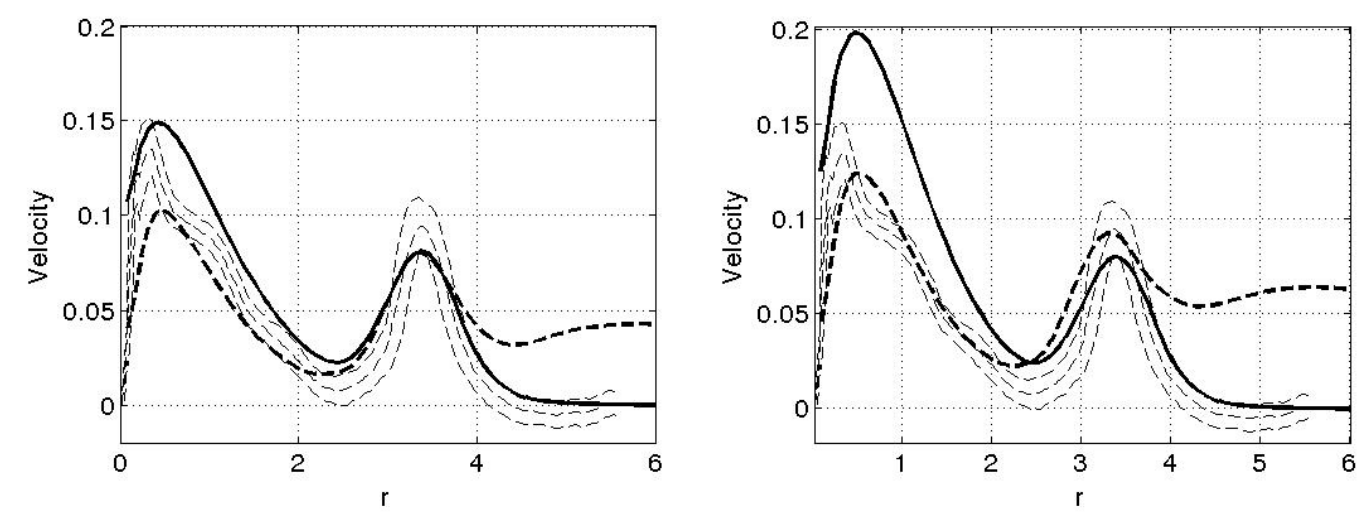

Figure 14. Superposition of the observed zonal velocity profile with error margins (thin dashed), the model zonal velocity profile used in initialization of numerical simulations (solid), and zonal velocity profile after the hexagon formation (dashed). In the right panel the amplitude of the initial vortex was taken deliberately higher $(\epsilon=0.2)$ than in the right panel $(\epsilon=0.15)$, in order to arrive to a close to observations end state.

which is beyond the scope of this paper.

The same tendency is observed in the distribution of zonally averaged angular momentum represented in Fig. 15 by its deviation from the initial distribution. The angular momentum of the system is practically conserved (note that we have numerical dissipation and sponges at the boundaries). Fig. 15 gives an idea of angular momentum fluxes which are represented with the help of the difference of 

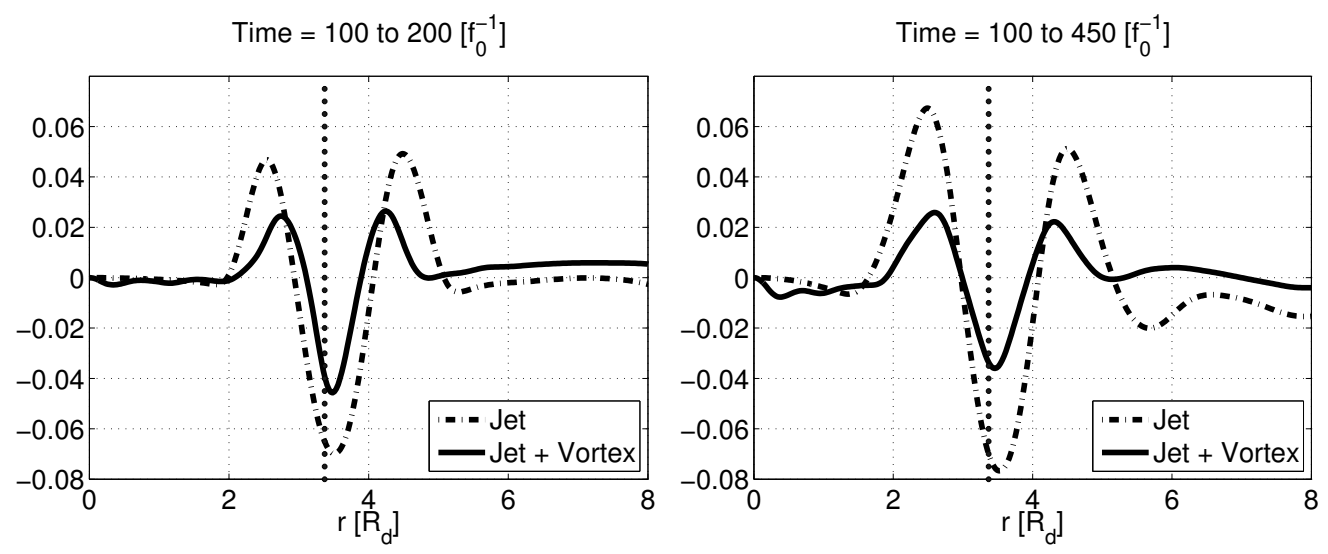

Figure 15. Difference of zonally averaged angular momentum density per unit mass $M$ of "jet" and "jet+vortex" configurations at times $t=200 f_{0}^{-1}$ (left panel), and $t=450 f_{0}^{-1}$ (right panel), and time $t=100 f_{0}^{-1}$, in the northern hemisphere. Vertical dashed line represents the initial maximum of of the jet velocity.

$M(t)-M\left(t_{0}\right)$ the angular momentum density per unit mass $M$

$$
M(r)=r v+f \frac{r^{2}}{2}
$$

at a given time $t$ and a fixed time $t_{0}$. We have chosen $t_{0}=100 f_{0}^{-1}$ to avoid oscillations due to emission of inertia-gravity waves, and their partial reflection by (imperfect) sponges, at the initial stages of the evolution. The Figure shows a net angular momentum flux from the polar vortex to the jet, which diminishes in time in the jet+vortex configuration, but continues to act in the jet-only one.

A tentative interpretation of the stabilization of the hexagonal structure of the circumpolar jet is that the central vortex is close enough to sweep the inner secondary vortices appearing due to development of the barotropic instability of the jet, and thus damp the meandering observed in the "jet-only" case.

The above-described results correspond to initialization with a single dominant mode $l=6$. We carried out simulations initialized with a spectrum of randomphase modes with different $l=2,3, \ldots, 8$, which also leads to a hexagonal jet with similar properties as in the reference case of single-mode initialization, although it takes a longer time to settle. Fig.16 illustrates the emergence of $l=6$ as the dominant mode in this case. 


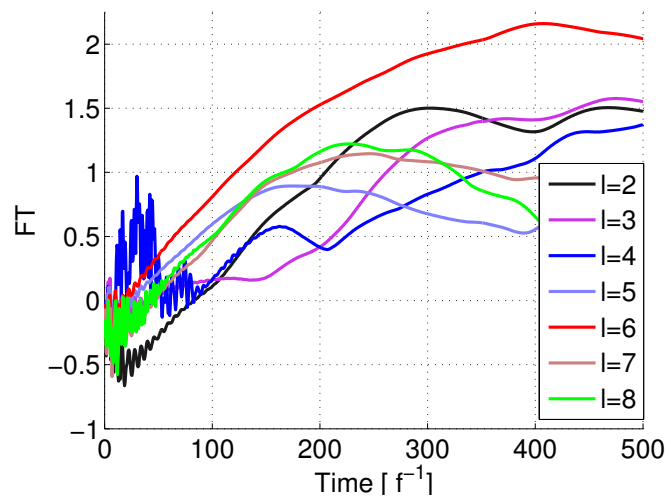

Figure 16. Evolution of the logarithms of the normalized amplitudes of the Fourier modes of the azimuthal velocity in a simulation of "jet+vortex" configuration in the northern hemisphere initialized with modes $l=2,3, \ldots, 8$ with the same (small) amplitudes and random phases. $V(r)$ is the same as Fig.1.

\section{Summary and discussion}

In the present paper we used the RSW model and the state-of-the-art Cassini observations of the polar hexagonal jet and central vortex (Antunano et al., 2015) for a search of dynamical mechanisms that could explain the existence and longevity of the Saturn's northern polar hexagon and the absence of its counterpart at the South pole. Insufficient knowledge of the vertical structure of the Saturn's atmosphere justifies the use of such vertically integrated model, where all information about the vertical structure is condensed in a single parameter, the Rossby deformation radius $R_{d}$. We used a fit of the observed velocity distributions to perform detailed linear stability analyses and to initialize nonlinear RSW simulations. In contrast with existing literature we analyzed not only a "jet-only", but also a "jet+vortex" configuration, and showed the importance of including the central vortex in the system.

Our main results are as follows

(1) There indeed exists a region of parameters where the most unstable mode of the jet-only configuration has azimuthal wavenumber $l=6$, although small changes of parameters may shift this value up or down. For the distance actually observed between the northern polar jet and the North Pole, the azimuthal wavenumber $l=6$ is obtained for a Rossby radius of deformation $R_{d} \approx 3200 \mathrm{~km}$.

(2) In the Southern hemisphere configuration, the most unstable mode has an azimuthal wavenumber higher than 8 , with a precise value depending on $R_{d}$. 
This is related to the distance of the southern polar jet from the South pole being larger than in the Northern hemisphere.

(3) The preceding results hold for both "jet-only" and "jet+vortex" cases. The difference between the two configurations is revealed by high-resolution fully nonlinear simulations initialized either with the most unstable mode of small amplitude, or with a random combination of unstable modes, superimposed onto the background velocity profile. In the "jet-only" configuration, nonlinear saturation of the instability leads to the formation of counter-jets and strong distortion of the hexagon at the late stages of the evolution, consistently with the standard scenario of saturation of the barotropic instability. On the contrary, in the "jet+vortex" configuration, the central vortex stabilizes the hexagon, leading at the late stages of the evolution to a configuration close to the observed one.

The persistence of the hexagonal structure in our RSW simulations suggests that the combination of central vortex and hexagonal jet structure could be an exact solution to the system. Proving this hypothesis is a challenge. Such proof would close the problem because, however robust and long-lived is this structure in our direct numerical simulations, although these latter are very long by the standards of computational fluid dynamics (more than a thousand of inertial periods), they remain far short of the time of observations of the Saturn's hexagon.

Compared to the existing literature, although the model and the background flow configurations are different, our "jet-only" configuration yields a "vortex-street" unstable northern polar hexagon similar to the results of Morales-Juberias et al. (2011), and the "jet+vortex" configuration yields a "meandering jet" stable northern polar hexagon, similar to Morales-Juberias et al. (2015), featuring the sharp straight-line PV gradients on the six sides of the hexagon. This means that the presence of the central vortex could stabilize the northern polar hexagonal jet already in a simple barotropic model, while a (baroclinic) vertical shear was necessary in Morales-Juberias et al. (2015) for stabilization of the hexagonal "meandering jet".

In spite of successful representation of the hexagon and explanation of its absence at the opposite pole, we still have two caveats in our scenario. The first one is too high phase velocity of the hexagon. We present in Table 1 the values of the angular (or phase) velocity $c$ of the hexagon for different values of parameters, as follows from the linear stability analysis of both the "jet-only" and the "jet+vortex" configurations (it should be stressed that the angular velocities retrieved from the direct nonlinear RSW simulations are close to results of the linear stability analysis). In both cases, the hexagon in our model is not quasi-stationary 
Table 1

Phase velocity of the hexagon

\begin{tabular}{|c|c|c|c|}
\hline$\epsilon$ & $R_{d}[\mathrm{~km}]$ & $c \quad\left[\mathrm{rad} . f_{0}\right]$, sole jet & $c \quad\left[\mathrm{rad} . f_{0}\right]$, jet+vortex \\
\hline 0.08 & 3200 & $7.33 \times 10^{-3}$ & $7.83 \times 10^{-3}$ \\
\hline 0.09 & 3200 & $8.40 \times 10^{-3}$ & $8.88 \times 10^{-3}$ \\
\hline 0.10 & 3200 & $9.38 \times 10^{-3}$ & $9.88 \times 10^{-3}$ \\
\hline 0.11 & 2400 & $7.33 \times 10^{-3}$ & $7.51 \times 10^{-3}$ \\
\hline 0.12 & 2400 & $7.71 \times 10^{-3}$ & $8.20 \times 10^{-3}$ \\
\hline 0.14 & 2400 & $9.06 \times 10^{-3}$ & $9.56 \times 10^{-3}$ \\
\hline
\end{tabular}

as in the observations (Sánchez-Lavega et al., 2014) $(\omega=+0.0128 \pm 0.0013$ degree longitude per Saturn day), but is rotating with $c \simeq+8 \times 10^{-3}$ radian. $f_{0}$, i.e. $\omega=+6$ degree longitude per Saturn day, meaning that the hexagonal pattern achieves a complete rotation in Saturn's rotating frame in 60 days, which is much higher than in observations. Sánchez-Lavega et al. (2014) use, however, Voyager's System III reference frame based on Saturn's Kilometric Radiation (SKR). Not only Cassini's SKR measurements yield a 8-min longer rotation period with respect to Voyager's (Gurnett et al., 2007), but alternative methods based on gravity measurements (Helled et al., 2015), or potential vorticity mapping (Read et al., 2009b), yield a 5-min to 7-min smaller rotation period, with respect to Voyager's. Assuming our shallow-water simulations are consistent with the atmospheric dynamics framework adopted in Read et al. (2009b), which entails that our simulated $+6^{\circ} /$ day drift rate would translate to a slower $+3^{\circ} /$ day drift rate in the Voyager SKR reference, and even a drift rate close to $0^{\circ} /$ day in the Cassini SKR reference.

The second one is the exaggerated zonal velocity in low latitudes resulting from the saturation of the instability. The second caveat is most probably due to the limitations of the gamma-plane model, simulations with the RSW model on the full sphere would be sufficient to answer the question whether this is a defect of the model, or of the gamma-plane approximation. The first one can be probably resolved by introducing a vertical structure in the model, such as an active lower layer, although this would require a number of ad-hoc hypotheses which we are reluctant to introduce. We should recall that baroclinic waves are slower than the barotropic ones, so a baroclinic structure would necessary slow down the system, the quantitative estimates depending on the details of the vertical structure. 
It should be also emphasized that we were working with a dissipationless version of the model. One of the reasons was to reduce the number of ad hoc hypotheses. Of course, an explicit (turbulent) viscosity and diffusion terms could be added in the r.h.s. of momentum and mass conservation equations of the model. In order to maintain any non-trivial steady state a forcing should be also added in this case to prevent dissipative decay. Both forcing and dissipation (the values of turbulent viscosity and diffusivity) would be necessarily ad hoc and should be adjusted to produce the observed mean flow. Above, we were pursuing the idea that the Saturn's polar hexagon results from an instability of this mean flow, i. e. the polar jet plus polar vortex system. The only difference, if this system is considered as a solution of forced-dissipative equations instead of non-forced non-dissipative ones, is that eigen-frequencies of its small perturbations would be subject to additional viscous damping, which is necessarily weak in view of longevity of the structure in question. A radiative damping can be also introduced in the system. In shallow-water modeling of the atmosphere such damping is usually represented by relaxation of the thickness of the layer towards some radiative equilibrium value with some characteristic relaxation time. As this time is presumably very long for Saturn, it was neglected. Another reason of working with inviscid model is that numerical scheme used for simulations of the saturation of the instability is practically dissipationless, which allows for very long-time runs.

\section{A Influence of moist convection upon the devloping instability}

Radiative damping mentioned above is an example of diabatic flux in the system. Another one, which was recently invoked for explanation of the emergence of Saturn's polar vortex with the help of the RSW model (O'Neill et al., 2016), and is recurrently evoked to explain the maintenance of alternating jets on giant planets, cf. e.g. (Showman, 2007) is moist convection. In relation to the discussion above, the moist waves are slower than the "dry" ones, so the moist effects could, in principle, explain the observed slowness of the hexagon's rotation. The condensation and moist convection can be included in the RSW model in a self-consistent way (Bouchut et al., 2009). For this, a new quantity, the bulk moisture content in the atmospheric column $Q$ is introduced. It is conserved in the absence of phase transitions, but acquires a sink if condensation is switched on. The condensation happens whenever the threshold of saturation $Q^{s}$ is crossed. An extra convective flux through the upper surface of the atmospheric layer is added and linked to the latent heat of condensation. The resulting equations of so-called moist-convective 

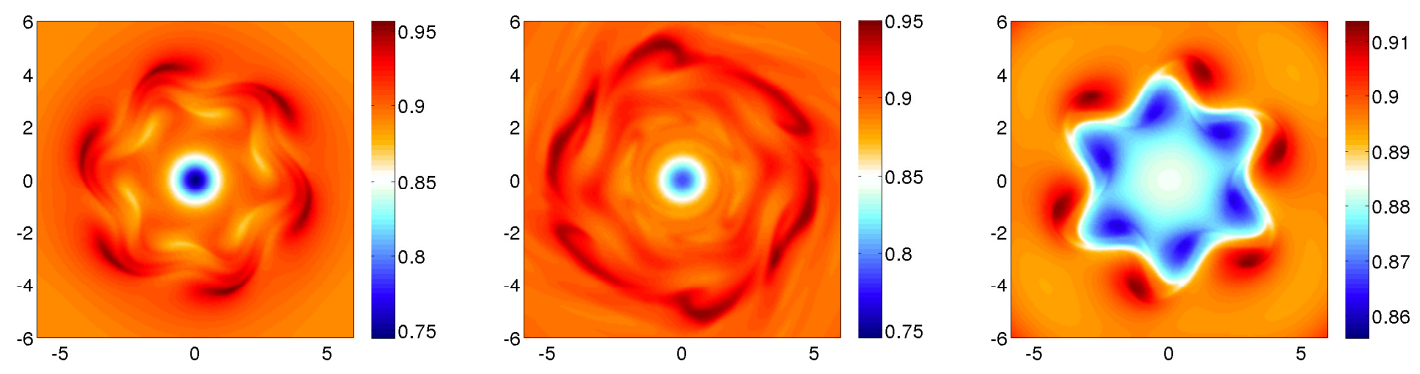

Figure 1. Snapshots of humidity initialized with uniform through the whole domain value $q_{0}=0.89$ corresponding to the "jet+vortex" (left and middle panel at $t=150,1000 f_{0}{ }^{-1}$ ) and "jet-only" (right panel at $t=150 f_{0}{ }^{-1}$ ) configurations.

rotating shallow water (mcRSW) read:

$$
\left\{\begin{array}{l}
\partial_{t} \boldsymbol{v}+(\boldsymbol{v} \cdot \boldsymbol{\nabla}) \boldsymbol{v}+f \hat{\boldsymbol{z}} \times \boldsymbol{v}=-g \boldsymbol{\nabla} h \\
\partial_{t} h+\boldsymbol{\nabla} \cdot(\boldsymbol{v} h)=-\beta P \\
\partial_{t} Q+\boldsymbol{\nabla} \cdot(Q \boldsymbol{v})=-P
\end{array}\right.
$$

with a coefficient $\beta$ related to background stratification, and a simple relaxation parameterization for condensation $P$ with relaxation time $\tau$ :

$$
P=\frac{Q-Q^{s}}{\tau} H\left(Q-Q^{s}\right)
$$

These new elements can be easily incorporated in the finite-volume numerical scheme we are using.

Before making simulations with the full-strength mcRSW, we can get useful insights from analysing the evolution of $Q$ in the absence of the condensation sink. $Q$ then obeys the conservation equation, the third equation in (A.1) with $P \equiv 0$. The results of previous RSW simulations with added passive tracer $Q$ evolving from initially uniform distribution are presented in Fig. 1, for both "jet-only" and "jet+vortex" configurations. Fig. 1 shows that the excess of humidity resulting from the evolution of the system is concentrated in the vicinity of the jet, and does not affect the central vortex. It is generally known, and confirmed by the simulations with moist-convective RSW in Lambaerts et al. (2011); Rostami and Zeitlin (2017), that condensation and related latent heat release lead to intensification of the cyclones and damping of the anticyclones So we can infer that in the absence of additional sources of moisture, condensation would not help to maintain the central vortex, and thus to increase the longevity of the system, but would only impact the hexagonal jet on its equatorward flank. Simulations with the full 


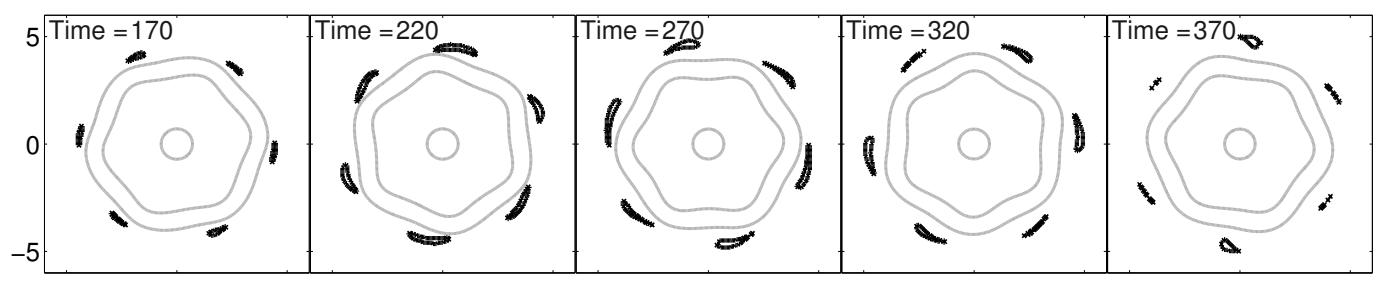

Figure 2. Snapshots of condensation (thick black contours) and isobars 0.6, 0.7 and 0.75 (grey contours) in a most-precipitating environment, as follows from the simulations with moist-convective RSW of Bouchut et al. (2009). Humidity is distributed uniformly in the flow domain, with initial value $Q=0.48$ with a saturation threshold at $Q_{s}=0.51$. Other initial or saturation values do not change the evolution qualitatively.

moist-convective RSW (A.1) confirm this prediction, as follows from Fig. 2 where evolution of condensation in time is displayed. Again, this result may change in the two-layer version of the model, but its introduction requires more information on the vertical structure of the Saturn's atmosphere.

Acknowledgments We are grateful to P. Read for stimulating discussions and encouragements, and to A. Antuñano (and colleagues) for providing the detailed Saturn's zonal velocity profiles described in their paper.

\section{References}

Aguilar, A. C. B., Read, P. L., Wordsworth, R. D., Salter, T., Yamazaki, Y. H., 2010. A laboratory model of saturn's north polar hexagon. Icarus 206 (2), 755 $-763$.

Allison, M., Godfrey, D. A., Beebe, R. F., 1990. A wave dynamical interpretation of saturn's polar hexagon. Science 247 (4946), 1061-1063.

Antunano, A., del Rio-Gaztelurrutia, T., Sanchez-Lavega, A., Hueso, R., 2015. Dynamics of saturn's polar regions. Journal of Geophysical Research: Planets 120 (2), 155-176.

Baines, K. H., Momary, T. W., Fletcher, L. N., Showman, A. P., Roos-Serote, M., Brown, R. H., Buratti, B. J., Clark, R. N., Nicholson, P. D., 2009. Saturn's north polar cyclone and hexagon at depth revealed by cassini/vims. Planetary and Space Science 57 (14-15), 1671 - 1681.

Bergmann, R., Tophoj, L., Homan, T., Hersen, P., Andersen, A., Bohr, T., 2011. Polygon formation and surface flow on a rotating fluid surface. Journal of fluid mechanics 679, 415-431.

Bouchut, F., 2007. Chapter 4 efficient numerical finite volume schemes for shallow water models. In: Zeitlin, V. (Ed.), Nonlinear Dynamics of Rotating Shallow 
Water: Methods and Advances. Vol. 2 of Edited Series on Advances in Nonlinear Science and Complexity. Elsevier Science, pp. 189 - 256.

Bouchut, F., Lambaerts, J., Lapeyre, G., Zeitlin, V., Nov. 2009. Fronts and nonlinear waves in a simplified shallow-water model of the atmosphere with moisture and convection. Physics of Fluids 21 (11), 116604-116604.

Boyd, J. P., May 1987. Orthogonal rational functions on a semi-infinite interval. J. Comput. Phys. 70 (1), 63-88.

Dowling, T. E., 1995. Dynamics of jovian atmospheres. Ann. Rev. Fluid Mech. 27, 293-334.

Godfrey, D., 1988. A hexagonal feature around saturn's north pole. Icarus 76 (2), $335-356$.

Gurnett, D. A., Persoon, A. M., Kurth, W. S., Groene, J. B., Averkamp, T. F., Dougherty, M. K., Southwood, D. J., 2007. The variable rotation period of the inner region of saturn's plasma disk. Science 316 (5823), 442-445.

Helled, R., Galanti, E., Kaspi, Y., 2015. Saturn's fast spin determined from its gravitational field and oblateness. Nature 520, 202-204.

Jansson, T. R. N., Haspang, M. P., Jensen, K. H., Hersen, P., Bohr, T., May 2006. Polygons on a rotating fluid surface. Phys. Rev. Lett. 96, 174502.

Lahaye, N., Zeitlin, V., Jan. 2015. Centrifugal, barotropic and baroclinic instabilities of isolated ageostrophic anticyclones in the two-layer rotating shallow water model and their nonlinear saturation. Journal of Fluid Mechanics 762, 5-34.

Lambaerts, J., Lapeyre, G., Zeitlin, V., 2011. Moist versus dry barotropic instability in a shallow-water model of the atmosphere with moist convection. Journal of the Atmospheric Sciences 68 (6), 1234-1252.

Liu, J., Schneider, T., 2015. Scaling of off-equatorial jets in giant planet atmospheres. Journal of the Atmospheric Sciences 72 (1), 389-408.

Marcus, P. S., Lee, C., 1998. A model for eastward and westward jets in laboratory experiments and planetary atmospheres. Physics of Fluids 10 (6).

Morales-Juberias, R., Sayanagi, K. M., Dowling, T. E., Ingersoll, A. P., 2011. Emergence of polar-jet polygons from jet instabilities in a saturn model. Icarus 211 (2), $1284-1293$.

Morales-Juberias, R., Sayanagi, K. M., Simon, A. A., Fletcher, L. N., Cosentino, R. G., 2015. Meandering shallow atmospheric jet as a model of saturn's northpolar hexagon. The Astrophysical Journal Letters 806 (1), L18.

O’Neill, M. E., Emanuel, K. A., Flierl, G. R., 2016. Weak jets and strong cyclones: Shallow-water modeling of giant planet polar caps. Journal of the Atmospheric Sciences 73 (4), 1841-1855.

Read, P., Conrath, B., Fletcher, L., Gierasch, P., Simon-Miller, A., Zuchowski, L., 2009a. Mapping potential vorticity dynamics on saturn: Zonal mean circulation from cassini and voyager data. Planetary and Space Science 57 (14-15), 1682 1698. 
Read, P. L., Dowling, T. E., Schubert, G., 2009b. Saturn's rotation period from its atmospheric planetary-wave configuration. Nature 460, 608-610.

Ripa, P., 1990. General stability conditions for a multi-layer model. J. Fluid Mech. $222,119$.

Rostami, M., Zeitlin, V., 2017. Influence of condensation and latent heat release upon barotropic and baroclinic instabilities of vortices in rotating shallow water f-plane model. Geophysical \& Astrophysical Fluid Dynamics 111 (1), 1-31.

Sanchez-Lavega, A., Lecacheux, J., Colas, F., Laques, P., 1993. Ground-based observations of saturn's north polar spot and hexagon. Science 260 (5106), 329-332.

Sayanagi, K., Baines, K., Dyudina, L., Fletcher, L., Sanchez-Lavega, A., R.A., W., Oct. 2016. Saturn's Polar Atmosphere.

Scott, R. K., Polvani, L. M., 2008. Equatorial superrotation in shallow atmospheres. Geophysical Research Letters 35 (24), n/a-n/a.

Showman, A. P., 2007. Numerical simulations of forced shallow-water turbulence: Effects of moist convection on the large-scale circulation of jupiter and saturn. Journal of the Atmospheric Sciences 64 (9), 3132-3157.

Sánchez-Lavega, A., del Río-Gaztelurrutia, T., Hueso, R., Pérez-Hoyos, S., GarcíaMelendo, E., Antuñano, A., Mendikoa, I., Rojas, J. F., Lillo, J., BarradoNavascués, D., Gomez-Forrellad, J. M., Go, C., Peach, D., Barry, T., Milika, D. P., Nicholas, P., Wesley, A., 2014. The long-term steady motion of saturn's hexagon and the stability of its enclosed jet stream under seasonal changes. Geophysical Research Letters 41 (5), 1425-1431.

Trefethen, L. N., 2000. Spectral methods in matlab. SIAM.

Vasavada, A., Horst, S., Kennedy, M., Ingersoll, A., Porco, C. C., Del Genio, A. D., West, R. A., 2006. Cassini imaging of saturn: Southern hemisphere winds and vortices. J. Geoph. Res. (Planets) 111, E05004.

Vatistas, G. H., Wang, J., Lin, S., 1994. Recent findings on kelvin's equilibria. Acta Mechanica 103 (1), 89-102. 